\title{
Decreased in vivo $\alpha 2$ adrenoceptor binding in the Flinders Sensitive Line rat model of depression
}

\author{
Anne M. Landau a, b, *, Jenny-Ann Phan a, c, Peter Iversen a, Thea P. Lillethorup a, b, \\ Mette Simonsen ${ }^{\mathrm{a}}$, Gregers Wegener ${ }^{\mathrm{d}, \mathrm{e}}$, Steen Jakobsen ${ }^{\mathrm{a}}$, Doris J. Doudet ${ }^{\mathrm{a}, \mathrm{f}}$ \\ a Department of Nuclear Medicine and PET Center, Aarhus University Hospital, Nørrebrogade 44, Building 10G, Aarhus, 8000, Denmark \\ b Center of Functionally Integrative Neuroscience/MINDlab, Aarhus University, Nørrebrogade 44, Building 10G, Aarhus, 8000, Denmark \\ ${ }^{\mathrm{c}}$ Department of Biomedicine, Aarhus University, Wilhelm Meyers Allé 4, Aarhus 8000, Denmark \\ d Translational Neuropsychiatry Unit, Aarhus University, Skovagervej 2, Building 14J.1, Risskov, 8240, Denmark \\ e Centre for Pharmaceutical Excellence, School of Pharmacy (Pharmacology), North-West University, Privaatsak X6001, Potchefstroom, 2520, South Africa \\ ${ }^{\mathrm{f}}$ Department of Medicine/Neurology, University of British Columbia, 2221 Wesbrook Mall, Vancouver, BC, V6T 2B5, Canada
}

\section{A R T I C L E I N F O}

\section{Article history:}

Received 3 October 2014

Received in revised form

18 December 2014

Accepted 20 December 2014

Available online 7 January 2015

\section{Keywords:}

Depression

Flinders sensitive line

Noradrenaline

Positron emission tomography

Yohimbine

\begin{abstract}
A B S T R A C T
Depression is a debilitating heterogenous disorder and the underlying mechanisms remain elusive. Alterations in monoaminergic neurotransmission, including noradrenergic, have been implicated in the etiology of depression. Although depression is difficult to model in animals, the availability of animal models with face, predictive and construct validity permits more in-depth investigations resulting in a greater understanding of the disease. We investigated the role of noradrenaline (NA) and $\alpha 2$ adrenoceptors in vivo in a genetic model of depression, the Flinders Sensitive Line (FSL) rat. We determined baseline differences in NA receptor volume of distribution to $\alpha 2$ adrenoceptors in FSL, in comparison with two routinely used controls, Flinders Resistant Line (FRL) and Sprague-Dawley (SD) rats using positron emission tomography (PET) imaging and the carbon-11 labeled radioligand yohimbine. We demonstrate a $42-47 \%$ reduction in the binding of the tracer in the cortex, striatum, cerebellum, thalamus and pons of FSL rats compared to the two control groups. Our results suggest that the behavioral deficits expressed in the FSL depression model are associated with functional over-activity of the NA system.
\end{abstract}

๑) 2015 Elsevier Ltd. All rights reserved.

\section{Introduction}

According to the World Health Organization, clinical depression is estimated to debilitate 350 million people globally (Marcus et al., 2012). The core symptoms of depression are anhedonia, behavioral despair, changes in appetite, weight loss or weight gain, neuroendocrine disturbances, alterations in sleep architecture and anxietyrelated behaviors (American Psychiatric Association, 2013). The last decades have seen many advances in therapeutic recourses and outcomes but the etiology remains elusive and is likely highly heterogenous: while some presentations appear to have a more

\footnotetext{
* Corresponding author. Department of Nuclear Medicine and PET Center, Aarhus University Hospital, Nørrebrogade 44, Building 10G, Aarhus C, 8000, Denmark. Tel.: +457846 4378; fax: +4578461662.

E-mail addresses: annie@pet.auh.dk (A.M. Landau), jap@biomed.au.dk (J.-A. Phan), peteiver@rm.dk (P. Iversen), thealillethorup@clin.au.dk (T.P. Lillethorup), simonsen@pet.auh.dk (M. Simonsen), wegener@clin.au.dk (G. Wegener), steejako@rm.dk (S. Jakobsen), ddoudet@mail.ubc.ca (D.J. Doudet).
}

endogenous nature, without apparent behavioral causes, others appear triggered by the cumulative effects of traumatic life events. It is unknown if the underlying neurochemistry varies with individual behavioural phenotypes, e.g. if an individual presenting with anxiety, irritability or high level of alarm and activity state has the same neurotransmitter imbalances as individuals with hopelessness and sadness. Similarly, there is no uniform pathology: the depressive disorders are heterogenous, not associated with clear neuronal loss, and may stem more from a combination of genetic predisposition and environmental influences leading to neuronal dysfunction rather than obvious pathology (Kessler, 1997; Klengel and Binder, 2013). However, several decades of intense pharmacological research have demonstrated the contribution of monoaminergic transmission to the disease (Manji et al., 2001; Schildkraut, 1965).

Most effective pharmacological antidepressants affect to some degree one, and often more, of the main monoamines: serotonin (5HT), noradrenaline (NA) and dopamine (DA) (Marks et al., 2008). While the serotonin system in mood disorders has been subjected 
to scrutiny because of the empirical observation of the normalizing effects of most pharmacological antidepressants on serotonin levels, the relationship between $\alpha 2$ adrenergic receptors and therapeutic effects has been recognized for decades (Cohen et al., 1980; Cottingham and Wang, 2012), and yet, the status of the $\alpha 2$ adrenoceptor in depression remains unclear.

One of the limitations to the study of depression is the lack of appropriate animal models. There is no current adequate model of depression in animals that recapitulates the wide array of symptoms. Existing models use selected lesions, genetic manipulation, systemic or local administration of drugs to alter the function of specific neurotransmitters or brain regions, or application of prenatal/early natal or long-term behavioral stressors to produce some of the behavioral observations, but without achieving the complete picture. In depression, it is however possible to use partial models to answer focused questions, for example to study neuroplastic changes or the involvement of monoamines in etiology, progression or therapeutic usefulness.

Among the various depression/anxiety rat models, the Flinders sensitive line (FSL) has many advantageous features. This rat model was developed by the selective breeding of Sprague Dawley (SD) rats for increased responses to an anticholinesterase agent (Overstreet, 1986; Overstreet and Russell, 1982). The Flinders resistant line (FRL) demonstrates a behavioral response similar to that of the original SD and is often used as a control line in study of FSL animals. Behaviorally, FSL rats are less active in novel environments, display sleep disturbances, reduced saccharin preference, and increased responsiveness to stress eg. anhedonia-like symptoms or increased immobility in the forced swim test, features reversible by chronic, but not acute, treatment with antidepressant drugs (Overstreet et al., 2005). FSL rats have reduced serotonin synthesis in the raphe nuclei and limbic areas implicated in depression (Hasegawa et al., 2006). Furthermore, the behavior of FSL rats improves with tricyclic antidepressants, serotonin reuptake inhibitors (SSRI) and ECT therapy (Chen et al., 2010; JimenezVasquez et al., 2007). Interestingly, FSL rats also have lower vesicular monoamine transporter (VMAT2) in striatal and limbic areas (Schwartz et al., 2003). Taken together, the FSL rat is a genetic model of depression with good face and predictive validity (Overstreet et al., 2005; Overstreet and Wegener, 2013). However, while the role of NA in depression is well accepted, and desipramine is often used in this model as an antidepressant of choice (Roth-Deri et al., 2009; Zangen et al., 1999), no studies to date have directly investigated in vivo the role or status of NA and its receptors in FSL rats.

We have recently developed $\left[{ }^{11} \mathrm{C}\right]$ yohimbine, an antagonist of the $\alpha 2$ NA receptors, as a potential tracer for positron emission tomography (PET) brain imaging studies (Jakobsen et al., 2006). Yohimbine is a stimulant alkaloid naturally found in several plants. It has been used as a weight loss dietary extract and to treat sexual dysfunction as well as an adjunct to antidepressant therapy (Tam et al., 2001). Despite some antihypertensive properties, it generally increases blood pressure at rest, which is thought to be mediated via central antagonism of $\alpha 2$ adrenergic receptors (Biaggioni et al., 1994). Our earlier studies have found that in tracer concentrations, yohimbine exhibits high selectivity for $\alpha 2$ sites in vivo in pigs (Jakobsen et al., 2006) which is displaceable by amphetamine challenge (Landau et al., 2012a). Central $\alpha 2$ NA receptors are widely expressed pre-synaptically primarily on NA cell bodies and dendrites in the locus coeruleus (LC). They are also widely expressed, mostly post-synaptically in every projection area of the NA neurons, throughout the entire cerebrum. As a consequence, high specific activity yohimbine is a valuable tool to assess relative alterations in the regional distribution and density of central $\alpha 2$ receptors. In this study, we used $\left[{ }^{11} \mathrm{C}\right]$ yohimbine to investigate the differences in $\alpha 2$ adrenoceptor binding in the FSL rat model of depression compared to control FRL and SD rats.

\section{Materials and methods}

\subsection{Animals}

Adult female FSL and FRL rats from breeding colonies at the Centre for Psychiatric Research, and Sprague Dawley (SD) rats from Taconic were used in this study $(220-260 \mathrm{~g})$. The animals were pair-housed and the individuals were issued from different dams. The FSL and FRL animals were bred in-house in same rooms and conditions and the SD were purchased as young animals and spent several weeks in the same environment as the FSL and FRL prior to scanning. The animals were kept on a normal 12-h light/12-h dark cycle and given free access to food and water. The study protocol was approved and regulated by the Danish Committee on Ethics in Animal Experimentation (authorization number: 2007/561-1378) and all efforts were made to reduce the number of animals used in this study and to minimize suffering.

\subsection{MicroPET imaging}

The rats ( $\mathrm{N}=6$ per group) were initially anesthetized in an isoflurane (2\%) chamber. An arterial catheter was placed in the femoral artery. The rats were then positioned prone in a plastic stereotaxic frame designed to fit in the gantry of the tomograph (microPET R4, CTI, Concorde Microsystems). Anesthesia was maintained with $1.8-2 \%$ isoflurane delivered through a mask fitted to the head holder throughout the procedure. As yohimbine is a substrate for the p-glycoprotein transport system of the blood brain barrier in rodents (Pearce et al., 1989), a nonspecific p-glycoprotein inhibitor, cyclosporine-A (50 mg/kg IV) was administered $30 \mathrm{~min}$ prior to tracer injection to facilitate the penetration of the radioligand into the brain. After a 10 min transmission scan, the dynamic 90 min emission recording was initiated after bolus injection over $15-20 \mathrm{~s}$ of approximately $30-40 \mathrm{MBq}$ $(100-200 \mu \mathrm{L}$ volume; injected mass $0.06-0.8 \mu \mathrm{g})$ of high specific activity $\left[{ }^{11} \mathrm{C}\right]$ yohimbine. Arterial sampling was performed to determine the input function for data analysis about every 15 s during the first two minutes of the scan then at 3, 5, 10, $20,30,45,60,75$ and 88 min post tracer injection. Three drops of blood (about $150 \mu \mathrm{L}$ ) were sampled at each time and was replaced with an equivalent volume of sterile saline. Body temperature during the scans was maintained around 36-36.5 ${ }^{\circ} \mathrm{C}$ using a heat lamp.

\subsection{Analysis and statistics}

A decay-corrected plasma time activity curve was produced from the arterial samples obtained during the study. Attempt at measuring metabolites yielded inconclusive data in all animals, suggesting that yohimbine was poorly if at all metabolized in rats. As a result, only the total plasma time activity curve was used as the input function for measurement of the volume of distribution $\mathrm{V}_{\mathrm{T}}$. The $\mathrm{V}_{\mathrm{T}}$ was then divided by the free fraction in order to obtain the volume of distribution corrected for free plasma concentration $\left(\mathrm{V}_{\mathrm{T}} / f_{\mathrm{P}}\right)$, where $f_{\mathrm{P}}$ is the concentration of tracer in plasma that is not bound to plasma protein.

Determination of the plasma free fraction was done in a different set of SD, FRL and FSL rats. We elected to perform the measurements in a separate group of rats as, during the PET studies, plasma is already collected to construct the time activity curve and added sampling of the volume of plasma needed to adequately perform the free fraction measurements in triplicate could have significantly affected the animals' welfare as well as the acquisition of physiologically relevant data, due to potentially large decreases in blood volume and hematocrit. Thus, independent groups of isoflurane-anesthetized rats were processed in a manner similar as the animals used for the PET studies: they were injected with cyclosporine-A (50 mg/ $\mathrm{kg} \mathrm{IV}$ ) and $30 \mathrm{~min}$ later were bled through intra-cardiac puncture to obtain sufficient amount of whole blood. To determine the plasma free fraction, $f_{\mathrm{P}}$, measurement of plasma protein binding was performed using standard procedures as previously described (Gandelman et al., 1994). Briefly, the plasma and an equivalent amount of PBS were spiked with a small amount of $\left[{ }^{11} \mathrm{C}\right.$ yohimbine and incubated for $10-15 \mathrm{~min}$ at room temperature. Aliquots of $50 \mu \mathrm{L}$ of this solution were used to measure the total activity (unfiltered plasma) and the remaining volume was equally divided $\left(\sim 150 \mu \mathrm{L}\right.$ each) into three ultrafiltration devices (Centrifree ${ }^{\circledR}$ UF Device, Millipore, $30 \mathrm{kDa}$ molecular weight cut-off). After centrifugation for $20 \mathrm{~min}$ at $10,000 \mathrm{~g}$, aliquots of $50 \mu \mathrm{L}$ of the ultrafiltrate were removed to measure the free activity (filtered protein-free plasma). The activity in the ultrafiltrates, plasma and PBS solutions was counted in a gamma counter (Packard Cobra Gamma Counter, Model D5003) and decay-corrected. The data from the triplicate measurements were averaged. A correction factor for device recovery was calculated from the PBS data as C, the ratio of the total activity (unfiltered) in the buffer to the activity in the filtered buffer. The free fraction $f_{\mathrm{P}}$ of $\left[{ }^{11} \mathrm{C}\right]$ yohimbine was calculated as the ratio of the activity of the filtered ultrafiltrate to the activity of the unfiltered plasma multiplied by the correction factor $C$. The data from the individual animals were averaged to obtain a mean free fraction per group/strain.

MicroPET images were processed using Montreal Neurological Institute (MINC) software. Each scan was co-registered to an average rat brain atlas (Rubins et al. 2003). A set of regions of interest (ROI) (frontal cortex, striatum, thalamus, pons and cerebellum) was manually drawn on the atlas. This ROI template was applied to 
the registered PET data to produce regional time activity curves. The total volume of distribution $\left(\mathrm{V}_{\mathrm{T}}\right)$ for each region was obtained using the Logan graphical analysis (Logan et al., 1990) during the 30-90 min period of the scan, using each animal's plasma curve as the input function. The $\mathrm{V}_{\mathrm{T}}$ and $\mathrm{V}_{\mathrm{T}} / f_{\mathrm{P}}$ were analyzed using a two way ANOVA with STRAIN and REGION as the factors, followed by a Bonferroni correction using Graphpad Prism version 5 for Mac OSX (GraphPad software Inc, La Jolla, CA).

\section{Results}

The tracer plasma free fraction in FRL $(0.21 \pm 0.02$ mean \pm SD $)$ and SD $(0.21 \pm 0.02)$ rats was not significantly different. It was significantly reduced $(P=0.02)$ in the FSL animals $(0.17 \pm 0.02)$. Averaged plasma activity curves were constructed for each group from the data of each individual animal corrected by the amount of activity injected per $\mathrm{kg}$ of body weight. There were no differences in the plasma total time activity curves between the 3 groups $(\mathrm{p}=0.85, \mathrm{~F}=0.16)($ Fig 1$)$

Fig. 2 shows the group average time activity curves (normalized for injected activity per $\mathrm{kg}$ of body weight) for three cortical and subcortical alpha2 adrenoceptor binding regions (frontal cortex, striatum and thalamus) for all 3 groups. A three way ANOVA revealed no time $\times$ strain interaction for any region $(F=0.25$ to $0.44, \mathrm{Df}=32$, $\mathrm{p}>0.05$ ). The graph clearly shows the difference between the FSL animals and the 2 control groups in the different regions.

Two way ANOVA analysis of the $V_{T}$ showed a significant effect of REGION ( $\mathrm{F}=6.46$, Df $=4, \mathrm{p}=0.0002)$ and of STRAIN $(\mathrm{F}=54.03$, $\mathrm{Df}=2, \mathrm{p}<0.0001)$ but no interaction $(\mathrm{F}=0.41, \mathrm{Df}=8, \mathrm{p}=0.91)$. Post-Bonferroni correction of the group data revealed significantly lower $\mathrm{V}_{\mathrm{T}}$ in all the regions in the FSL rats compared with SD rats. There was no significant difference between SD and FRL animals.

Two way ANOVA analysis of the $\mathrm{V}_{\mathrm{T}} / \mathrm{f}_{\mathrm{P}}$ also demonstrated a significant effect of STRAIN $(\mathrm{F}=33.57, \mathrm{Df}=2, \mathrm{p}<0.0001)$ and REGION $(\mathrm{F}=6.7, \mathrm{Df}=4, \mathrm{p}=0.0001)$ and no interaction $(\mathrm{F}=0.29, \mathrm{Df}=8$, $\mathrm{p}=0.97$ ). Comparisons between STRAIN groups revealed statistically significant differences between the FSL and SD/FRL rats with FSL rats showing lowest $\mathrm{V}_{\mathrm{T}} / \mathrm{f}_{\mathrm{p}}$ binding.

Table 1 compares the average values of $V_{T}$ and $V_{T} / f_{P}(S E M)$ for all 3 groups in all 5 regions. Fig. 3 shows the $V_{T} / f_{P}$ for all 3 groups. Fig. 4 shows a co-registered parametric PET/MRI image of the $V_{T} / f_{P}$ of one rat in each of the three groups.

\section{Discussion}

In this study, we demonstrated a reduced binding of yohimbine in FSL rats compared to FRL and SD control rats. This observation is consistent with a previous study demonstrating a two to threefold

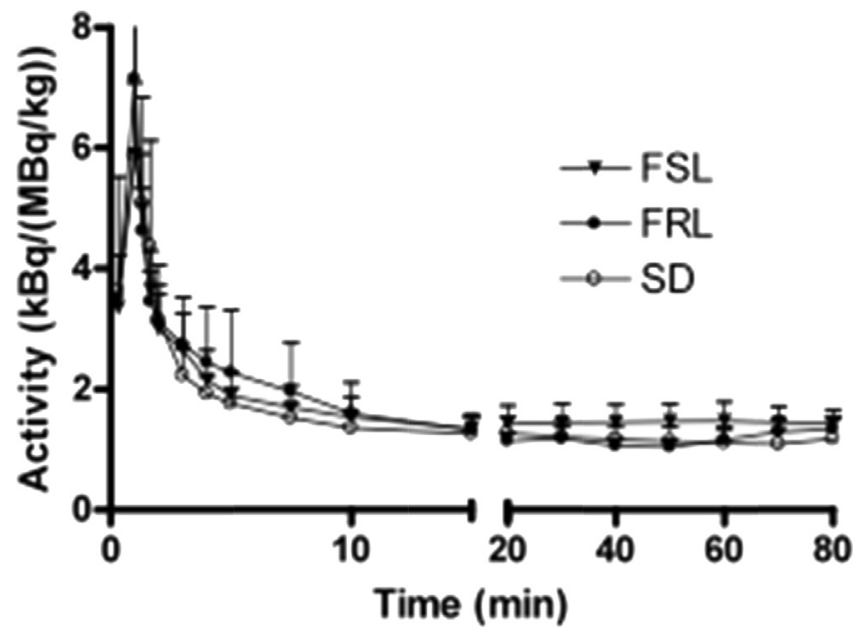

Fig. 1. Averaged total plasma activity curves in the 3 groups $( \pm$ SEM $)$. The early part of the curve was expanded to more clearly show the similarity between curves.
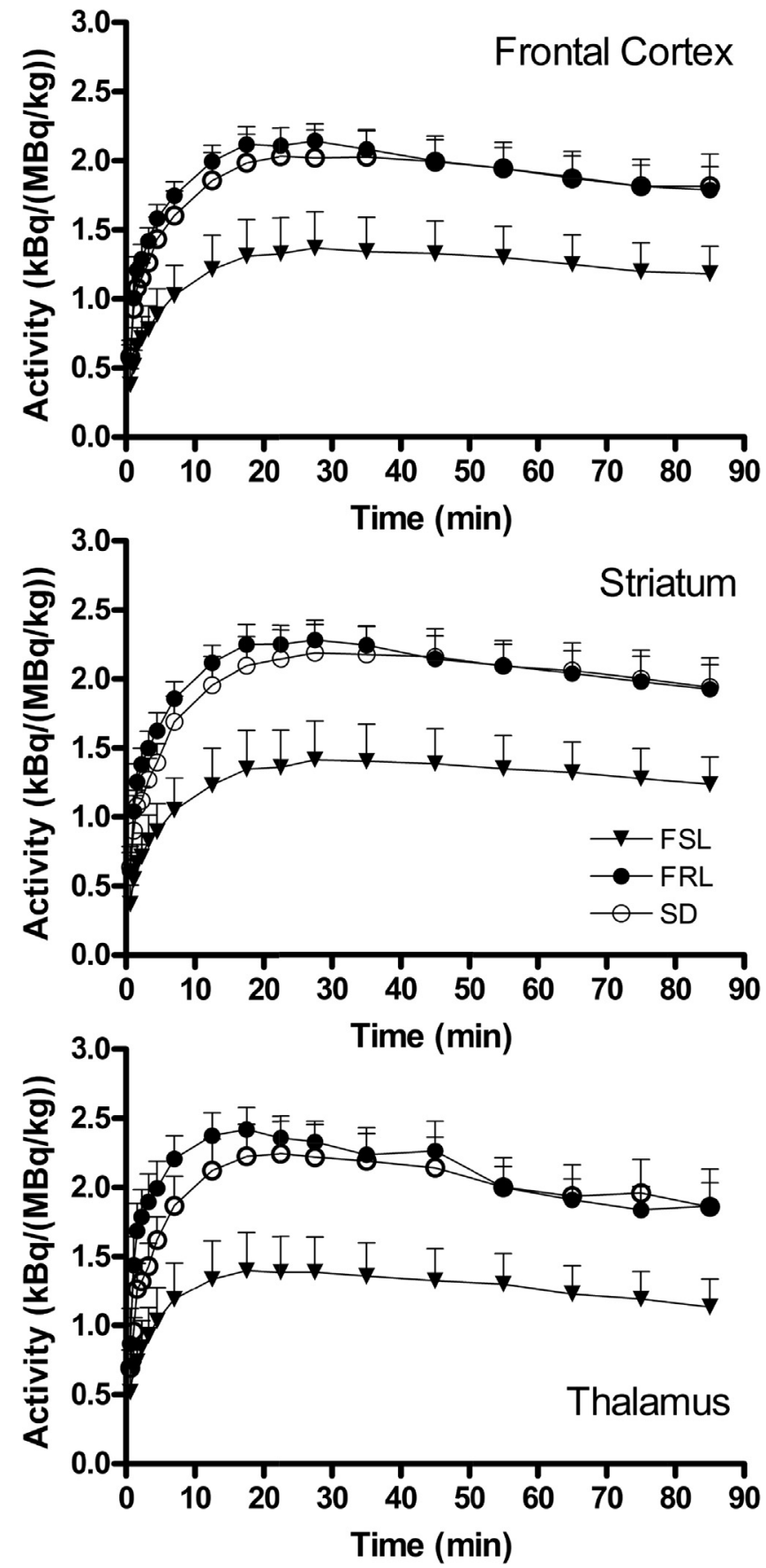

Fig. 2. Examples of averaged time activity curves in frontal cortex, striatum and thalamus in the 3 groups of animals. Activity is corrected for the amount of activity injected by $\mathrm{kg}$ of body weight $( \pm \mathrm{SEM})$. The similar shape of the curves graphically demonstrates the lack of difference in perfusion kinetics.

increase in NA levels in the nucleus accumbens, prefrontal cortex and hippocampus in FSL rats compared to SD rats (Zangen et al., 1999). Indeed, we have previously demonstrated that yohimbine is highly sensitive to changes in endogenous NA (Landau et al.,

Table 1

Volume of distribution $V_{T}$ and free-fraction corrected volume of distribution $V_{T} / f_{P}$ averaged across the 5 regions. Data shown ${ }_{ \pm}$SEM. The FSL column also shows the percent decrease in FSL binding compared to SD.

\begin{tabular}{llll}
\hline Units: $\left(\mathrm{mL} / \mathrm{cm}^{3}\right)$ & $\mathrm{SD}$ & FRL & FSL \\
\hline $\mathrm{V}_{\mathrm{T}}$ & $1.70 \pm 0.09$ & $1.71 \pm 0.1$ & $0.76 \pm 0.04(55 \%)$ \\
$\mathrm{V}_{\mathrm{T}} / \mathrm{f}_{\mathrm{P}}$ & $8.10 \pm 0.42$ & $8.13 \pm 0.47$ & $4.48 \pm 0.24(44 \%)$ \\
\hline
\end{tabular}




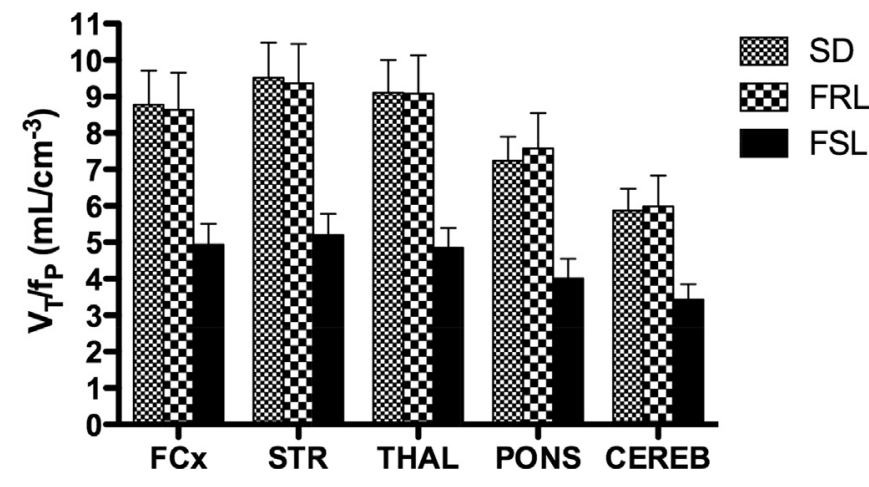

Fig. 3. Graphical representation of the volume of distribution corrected by the free plasma fraction values $\left(\mathrm{V}_{\mathrm{T}} / \mathrm{f}_{\mathrm{P}}\right)$ in the control Sprague Dawley (SD), Flinders Resistant Line (FRL) and Flinders Sensitive Line (FSL) rats. Error bars indicate standard error.

2012b; Phan et al., in press), probably through a competition process similar to what is hypothesized for raclopride, a tracer of the DA D2 receptor routinely used as a surrogate marker of DA release (Laruelle, 2000). Decreased in vivo yohimbine binding would thus reflect this heightened endogenous synaptic NA concentrations and/or receptor down-regulation.

Initial studies demonstrated that, in rodents, yohimbine is a substrate for the ABC-cassette family of blood brain barrier transport systems that include P-glycoprotein, and control and reduce entry of drugs into brain parenchyma. Differences between the human and rodent isoforms (Zolnerciks et al., 2011) may account for species variation as yohimbine is not a P-glycoprotein substrate in human. Cyclosporine-A is a commonly used non-specific Pglycoprotein inhibitor that has often been used in rodents and primates to overcome low entrance of PET tracers into the brain. Administered prior to the tracer, it permitted the acquisition of images allowing reliable regional identification and data quantification of yohimbine binding.

We were unable to reliably detect plasma metabolites in any of the 3 groups, SD, FRL or FSL despite testing of several HPLC methods and columns. Nevertheless, the HPLC traces were remarkably similar between animals, leading us to conclude that there was little or no significant measurable metabolism of the tracer. Thus, we used the total plasma activity curve as the input to evaluate $\mathrm{V}_{\mathrm{T}}$. The plasma free fraction $f_{p}$ was significantly lower in FSL rats compared to the FRL and SD controls. The reason for the difference in free fraction is unclear. A non-specific pharmacological drug like cyclosporine may have affected systemic pharmacokinetic factors and produced tracer-drug interactions independently of its effects on P-glycoprotein. This family of pharmacological agents is known to interact with metabolic functions, including liver enzymes. Kotsovolou et al. demonstrated significant differences in the expression of several major drug-metabolizing enzymes, notably in the CYP2 and CYP3 families of enzymes in FSL rats (Kotsovolou et al., 2010), which play a significant role in metabolizing numerous clinically used drugs, such as yohimbine and mirtazapine, another $\alpha 2$ adrenergic antagonist. While all the rats received cyclosporine, interactions of an unknown nature may have altered the systemic pharmacokinetics of the tracer in FSL rats, a strain bred over generations to express specific behaviors and in which the genetic makeup is not fully understood. The lack of difference in total plasma activity between strains with a decreased free fraction in FSL animals suggests a decrease in the concentration of circulating free tracer in these rats, supported by the clear difference in the brain time activity curves in every brain region in the FSL compared to the control rats. The similarity of the shape of the curves between strains argues for an unaffected mechanism of

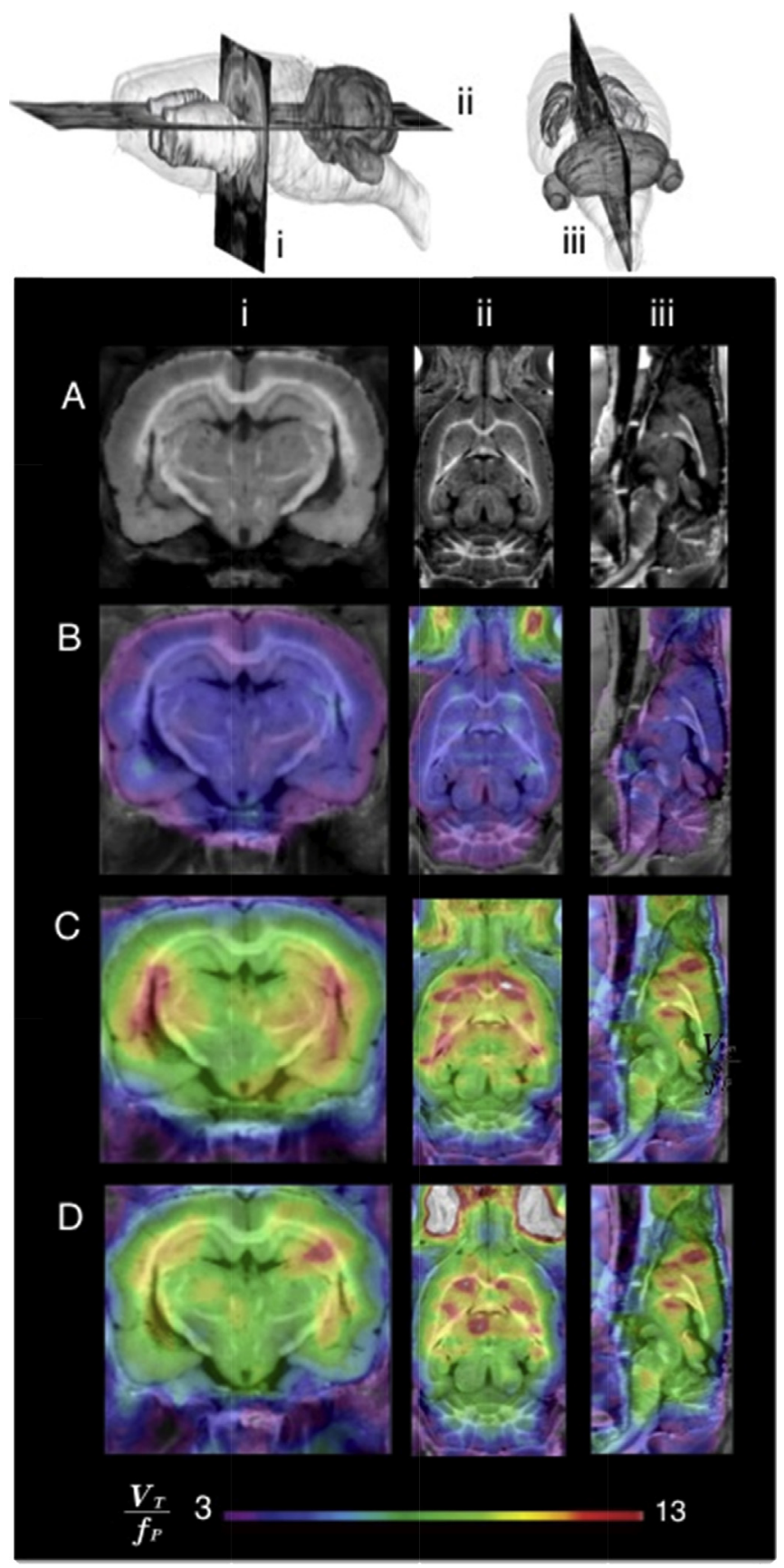

Fig. 4. Coronal (i), axial (ii) and (iii) sagittal parametric PET images superimposed on an MRI atlas (A) of the $V_{\mathrm{T}} / f_{\mathrm{P}}$ of a representative FSL (B), FRL (C) and SD (D) rat. Note the reduced $\mathrm{V}_{\mathrm{T}} / f_{\mathrm{P}}$ in the FSL rat compared to the control groups.

brain entrance and little effect of blood flow on the differences between FSL and controls. The differences in binding between the control groups and FSL rats remained highly significant even after correcting the volume of distribution $\mathrm{V}_{\mathrm{T}}$ by the tracer plasma free fraction $f_{\mathrm{P}}$.

A large section of the clinical literature on mood disorders strongly supports $\alpha 2$ receptor up-regulation as a result of dysfunction and/or loss of NA inputs from the LC and other brainstem and pontine nuclei (Ressler and Nemeroff, 1999). Increased density of $\alpha 2$ adrenoceptors and decreased density of NA transporters are observed in the LC (Ordway et al., 2003), suggesting NA neuron loss. Receptor binding studies in the brains of suicide 
victims demonstrate increased $\alpha 2$ adrenoceptor binding and receptor mRNA levels (Escriba et al., 2004; Gonzalez-Maeso et al., 2002), and increased receptor agonist binding in the hippocampus and cerebral cortex (Gonzalez et al., 1994). Similarly, a preliminary in vitro autoradiography study in different groups of female SD, FRL and FSL animals using $\left[{ }^{3} \mathrm{H}\right]-\mathrm{RX} 821002$, another selective tracer of the $\alpha 2$ receptors, performed in our lab as part of another study (Lillethorup et al., submitted) revealed either no significant change (thalamus, hippocampus, amygdala) or $\alpha 2$ receptor up-regulation (cortical regions: 15-23\%) compared to control SD.

While the post-mortem data are in apparent contradiction with our in vivo data, it is important to remember that in many cases, post-mortem autoradiography data reflect different processes than in vivo data: most significantly, the tissue is washed of any remaining endogenous ligand as part of the autoradiography method and the data represent the number of available receptors, not their functional state. The PET data are, on the contrary, acquired in a live subject in which all regulatory mechanisms are ongoing and the decrease in yohimbine binding may thus reflect a state of functional hyper-noradrenergic release. This hypothesis is supported by the increased levels of extracellular NA found in vivo in FSL rats compared to SD (Zangen et al., 1999). Furthermore, although in vivo PET data is often influenced by the choice of anesthetic, we have previously shown that yohimbine binding is fairly insensitive to changes in blood flow (Alstrup et al., 2013).

Increased markers of hyper-noradrenergia are also reported in some human depressive presentations (Brunello et al., 2003; Wong et al., 2000) and high levels of NA are often associated with heightened anxiety (Baldwin, 2006; Charney et al., 1984). Interestingly, in some contexts, anxiety and depression have been found to co-exist in FSL animals (Overstreet et al., 2004).

In conclusion, one may thus hypothesize that FSL animals represent a model of the anxious/depressive type of depression characterized by a functional state of high central levels of NA release, especially in stressful situations (pre-scan handling for example). This would suggest that the FSL rat model, like many animal models, while presenting a typical rodent depressive behavioral phenotype, reflects only a subset of the physiological underpinnings of depression in the human population and that more than one type of noradrenergic dysregulation may underlie the variable expression of mood disorders and behavioral dysfunctions.

\section{Acknowledgments}

This work was supported by a grant from Th. Maigaards Eftf. Fru Lily Benthine Lunds Fond af 1.6.1978 to AML and by the Aarhus University Hospital Department of Nuclear Medicine and PET Center.

\section{References}

Alstrup, A.K., Landau, A.M., Holden, J.E., Jakobsen, S., Schacht, A.C., Audrain, H. Wegener, G., Hansen, A.K., Gjedde, A., Doudet, D.J., 2013. Effects of anesthesia and species on the uptake or binding of radioligands in vivo in the Gottingen minipig. Biomed. Res. Int. 2013, 808713.

American Psychiatric Association, 2013. Diagnostic and Statistical Manual of Mental Disorders: DSM-IV. American Psychiatric Association, Washington DC.

Baldwin, D.S., 2006. Serotonin noradrenaline reuptake inhibitors: a new generation of treatment for anxiety disorders. Int. J. Psychiatry Clin. Pract. 10 (Suppl. 2), $12-15$.

Biaggioni, I., Robertson, R.M., Robertson, D., 1994. Manipulation of norepinephrine metabolism with yohimbine in the treatment of autonomic failure. J. Clin. Pharmacol. 34, 418-423.

Brunello, N., Blier, P., Judd, L.L., Mendlewicz, J., Nelson, C.J., Souery, D., Zohar, J. Racagni, G., 2003. Noradrenaline in mood and anxiety disorders: basic and clinical studies. Int. Clin. Psychopharmacol. 18, 191-202.
Charney, D.S., Heninger, G.R., Breier, A., 1984. Noradrenergic function in panic anxiety. Effects of yohimbine in healthy subjects and patients with agoraphobia and panic disorder. Arch. Gen. Psychiatry 41, 751-763.

Chen, F., Madsen, T.M., Wegener, G., Nyengaard, J.R., 2010. Imipramine treatment increases the number of hippocampal synapses and neurons in a genetic animal model of depression. Hippocampus 20, 1376-1384.

Cohen, R.M., Campbell, I.C., Cohen, M.R., Torda, T., Pickar, D., Siever, L.J., Murphy, D.L., 1980. Presynaptic noradrenergic regulation during depression and antidepressant drug treatment. Psychiatry Res. 3, 93-105.

Cottingham, C., Wang, Q., 2012. alpha2 adrenergic receptor dysregulation in depressive disorders: implications for the neurobiology of depression and antidepressant therapy. Neurosci. Biobehav. Rev. 36, 2214-2225.

Escriba, P.V., Ozaita, A., Garcia-Sevilla, J.A., 2004. Increased mRNA expression of alpha2A-adrenoceptors, serotonin receptors and mu-opioid receptors in the brains of suicide victims. Neuropsychopharmacology 29, 1512-1521.

Gandelman, M.S., Baldwin, R.M., Zoghbi, S.S., Zea-Ponce, Y., Innis, R.B., 1994. Evaluation of ultrafiltration for the free-fraction determination of single photon emission computed tomography (SPECT) radiotracers: beta-CIT, IBF, and iomazenil. J. Pharm. Sci. 83, 1014-1019.

Gonzalez, A.M., Pascual, J., Meana, J.J., Barturen, F, del Arco, C., Pazos, A., GarciaSevilla, J.A., 1994. Autoradiographic demonstration of increased alpha 2adrenoceptor agonist binding sites in the hippocampus and frontal cortex of depressed suicide victims. J. Neurochem. 63, 256-265.

Gonzalez-Maeso, J., Rodriguez-Puertas, R., Meana, J.J., Garcia-Sevilla, J.A., Guimon, J., 2002. Neurotransmitter receptor-mediated activation of G-proteins in brains of suicide victims with mood disorders: selective supersensitivity of alpha(2A)adrenoceptors. Mol. Psychiatry 7, 755-767.

Hasegawa, S., Nishi, K., Watanabe, A., Overstreet, D.H., Diksic, M., 2006. Brain 5-HT synthesis in the Flinders Sensitive Line rat model of depression: an autoradiographic study. Neurochem. Int. 48, 358-366.

Jakobsen, S., Pedersen, K., Smith, D.F., Jensen, S.B., Munk, O.L., Cumming, P., 2006. Detection of alpha2-adrenergic receptors in brain of living pig with $11 \mathrm{C}$ yohimbine. J. Nucl. Med. 47, 2008-2015.

Jimenez-Vasquez, P.A. Diaz-Cabiale, Z Caberlotto, L., Bellido, I. Overstreet, D. Fuxe, K., Mathe, A.A., 2007. Electroconvulsive stimuli selectively affect behavior and neuropeptide Y (NPY) and NPY Y(1) receptor gene expressions in hippocampus and hypothalamus of Flinders Sensitive Line rat model of depression. Eur. Neuropsychopharmacol. 17, 298-308.

Kessler, R.C., 1997. The effects of stressful life events on depression. Annu. Rev. Psychol. 48, 191-214.

Klengel, T., Binder, E.B., 2013. Gene-environment interactions in major depressive disorder. Can. J. Psychiatry 58, 76-83.

Kotsovolou, O., Ingelman-Sundberg, M., Lang, M.A., Marselos, M., Overstreet, D.H., Papadopoulou-Daifoti, Z., Johanson, I., Fotopoulos, A., Konstandi, M., 2010. Hepatic drug metabolizing profile of Flinders Sensitive Line rat model of depression. Prog. Neuropsychopharmacol. Biol. Psychiatry 34, 1075-1084.

Landau, A.M., Doudet, D.J., Jakobsen, S., 2012a. Amphetamine challenge decreases yohimbine binding to alpha2 adrenoceptors in Landrace pig brain. Psychopharmacol. Berl. 222, 155-163.

Landau, A.M., Dyve, S., Jakobsen, S., Alstrup, A.K., Doudet, D., Gjedde, A., 2012b. Update: inhibition plots of [C-11]yohimbine binding yield consistent estimates of non-displaceable volumes of distribution in multiple tests. J. Cereb. Blood Flow Metab. 32, S36-S37.

Laruelle, M., 2000. Imaging synaptic neurotransmission with in vivo binding competition techniques: a critical review. J. Cereb. Blood Flow. Metab. 20, 423-451.

Logan, J., Fowler, J.S., Volkow, N.D., Wolf, A.P., Dewey, S.L., Schlyer, D.J., MacGregor, R.R., Hitzemann, R., Bendriem, B., Gatley, S.J., et al., 1990. Graphical analysis of reversible radioligand binding from time-activity measurements applied to [N-11C-methyl]-(-)-cocaine PET studies in human subjects. J. Cereb. Blood Flow. Metab. 10, 740-747.

Manji, H.K., Drevets, W.C., Charney, D.S., 2001. The cellular neurobiology of depression. Nat. Med. 7, 541-547.

Marcus, M., Yasamy, M.T., Van Ommeren, M., Chisholm, D., Saxena, S., 2012. Depression, a Global Public Health Concern. WHO Department of Mental Health and Substance Abuse.

Marks, D.M., Pae, C.U., Patkar, A.A., 2008. Triple reuptake inhibitors: the next generation of antidepressants. Curr. Neuropharmacol. 6, 338-343.

Ordway, G.A., Schenk, J., Stockmeier, C.A., May, W., Klimek, V., 2003. Elevated agonist binding to alpha2-adrenoceptors in the locus coeruleus in major depression. Biol. Psychiatry 53, 315-323.

Overstreet, D.H., 1986. Selective breeding for increased cholinergic function: development of a new animal model of depression. Biol. Psychiatry 21, 49-58.

Overstreet, D.H., Friedman, E., Mathe, A.A., Yadid, G., 2005. The Flinders Sensitive Line rat: a selectively bred putative animal model of depression. Neurosci. Biobehav. Rev. 29, 739-759.

Overstreet, D.H., Keeney, A., Hogg, S., 2004. Antidepressant effects of citalopram and CRF receptor antagonist CP-154,526 in a rat model of depression. Eur. J. Pharmacol. 492, 195-201.

Overstreet, D.H., Russell, R.W., 1982. Selective breeding for diisopropyl fluorophosphate-sensitivity: behavioural effects of cholinergic agonists and antagonists. Psychopharmacol. Berl. 78, 150-155.

Overstreet, D.H., Wegener, G., 2013. The flinders sensitive line rat model of depression-25 years and still producing. Pharmacol. Rev. 65, 143-155. 
Pearce, H.L., Safa, A.R., Bach, N.J., Winter, M.A., Cirtain, M.C., Beck, W.T., 1989. Essential features of the P-glycoprotein pharmacophore as defined by a series of reserpine analogs that modulate multidrug resistance. Proc. Natl. Acad. Sci. U. S. A. 86, 5128-5132.

Phan, J.A., Landau, A.M., Wong, D.F., Jakobsen, S., Nahimi, A., Doudet, D.J., Gjedde, A. 2014. Quantification of $\left[{ }^{11} \mathrm{C}\right.$ lyohimbine binding to $\alpha 2$ adrenoceptors in rat brain in vivo. J. Cereb. Blood Flow. Metab. http://dx.doi.org/10.1038/jcbfm.2014.225 (in press).

Ressler, K.J., Nemeroff, C.B., 1999. Role of norepinephrine in the pathophysiology and treatment of mood disorders. Biol. Psychiatry 46, 1219-1233.

Roth-Deri, I., Friedman, A., Abraham, L., Lax, E., Flaumenhaft, Y., Dikshtein, Y., Yadid, G., 2009. Antidepressant treatment facilitates dopamine release and drug seeking behavior in a genetic animal model of depression. Eur. J. Neurosci. 30, 485-492.

Rubins, D.J., Melega, W.P., Lacan, G., Way, B., Plenevaux, A., Luxen, A., Cherry, S.R., 2003. Development and evaluation of an automated atlas-based image analysis method for microPET studies of the rat brain. Neuroimage 20, 2100-2118.

Schildkraut, J.J., 1965. The catecholamine hypothesis of affective disorders: a review of supporting evidence. Am. J. Psychiatry 122, 509-522.
Schwartz, K., Yadid, G., Weizman, A., Rehavi, M., 2003. Decreased limbic vesicular monoamine transporter 2 in a genetic rat model of depression. Brain Res. 965, $174-179$.

Tam, S.W., Worcel, M., Wyllie, M., 2001. Yohimbine: a clinical review. Pharmacol. Ther. 91, 215-243.

Wong, M.L., Kling, M.A., Munson, P.J., Listwak, S., Licinio, J., Prolo, P., Karp, B. McCutcheon, I.E., Geracioti Jr., T.D., DeBellis, M.D., Rice, K.C., Goldstein, D.S. Veldhuis, J.D., Chrousos, G.P., Oldfield, E.H., McCann, S.M., Gold, P.W., 2000 Pronounced and sustained central hypernoradrenergic function in major depression with melancholic features: relation to hypercortisolism and corticotropin-releasing hormone. Proc. Natl. Acad. Sci. U. S. A. 97, 325-330.

Zangen, A., Overstreet, D.H., Yadid, G., 1999. Increased catecholamine levels in specific brain regions of a rat model of depression: normalization by chronic antidepressant treatment. Brain Res. 824, 243-250.

Zolnerciks, J.K., Booth-Genthe, C.L., Gupta, A., Harris, J., Unadkat, J.D., 2011. Substrate- and species-dependent inhibition of P-glycoprotein-mediated transport: implications for predicting in vivo drug interactions. J. Pharm. Sci. 100, 3055-3061. 